\title{
10 Años del Uso del Laser Endovenoso en La Argentina
}

Segura J. ${ }^{1}$, Feldfeber J. ${ }^{1}$, Kornbeg A. ${ }^{1}$, Aimé M. ${ }^{1}$, Musci D. ${ }^{1}$

${ }^{1}$ Argentina.

E-mail: jorgesegura@yahoo.com.ar
Segura J.; Feldfeber J.; Kornbeg A.; Aimé M.; Musci D. 2013. 10 Años del Uso del Laser Endovenoso en La Argentina, p.27. In: Bastos, Francisco Reis. V Simpósio Internacional de Flebologia [Blucher Medical Proceedings n.1 v.1]. São Paulo: Blucher, 2014 http://dx.doi.org/10.5151/medpro-flebo-SIF_9

\section{Introducción}

El uso del Laser de Di lodo en forma endolunminal de la vena safena interna, por intermedio de fibras ópticas, primero de 810 y luego de 980 nanómetros en nuestros grupos de trabajo para el tratamiento de la insuficiencia venosa superficial, constituyó un gran reto inicialemte, ante nuestros contemporáneos, porque se trataba de un método nuevo que venía a competir con la cirugía venosa convencional, clásica y tradicional, avalada por más de 100 años de experiencia. El nuevo método tuvo que sortear muchos escollos, primero la pregunta del porqué algo nuevo, si lo conocido es eficaz ? Al principio con la crossectomía agregada, que incluía la incisión en el pliegue inguinal, la disección por planos hasta llegar al cayado de la safena y el tratamiento del cayado safeno y de las afluentes insuficientes, esta táctica quirúrgica trajo aparejadas controversias, por el hecho de hacer o no lo crossectomía. Nosotros recién en los últimos dos años decidimos hacer de modo sistemático el procedimiento endoluminal ecoguiado sin corssectomía. A medida que fuimos ganando experiencia nos sentimos más afianzados con la técnica de Laser Endoluminal Percutáneo Ecoguiado ( LEPE).

\section{Material Y Métodos}

Utilizamos inicialemente Equipos Laser Di lodo de 810 nanómetros, y a posteriori Equipos Laser de 980 nanómetros. Nuestra experiencia con el Laser 1470, ha sido solamente en 4 casos. Las Fibras ópticas que utilizamos, al principio eran de 400 micrones, actualemente utilizamos las de 600 micrones. La descarga de energía pulsada Laser que usamos para el tratamiento de la vena safena interna es de 10 Watts en el cayado y tronco del muslo; de 8 Watts en el tronco de la pierna y de 4 Watts en las venas perforantes, las energías descriptas son las máximas que administramos para el tratamiento, pudiendo ser menores, de acuerdo al caso; así también mayores en casos de excepción. La entrega de energía para producir la ablación o destrucción para cerrar u ocluir la vena, la realizamos en tiempo real ecográfico intraoperatorio, comprobándola de modo simultáneo, es decir a medida que vamos retirando la fibra Laser, vamos comprobando la ablación de la venas Se han descripto signos ecográficos, todos a partir del primer signo del ojo egipcio descripto por Baylly o del ojo safeno interno,que se observa cuando la safena interna a nivel del muslo la vemos 
en un corte trasnversal, bajo la apariencia de un ojo, siendo la deflexión de la fascia superficial, la que conforma los dos párpados, el superior y el inferior. Los que describimos en la técnica Laser son el del ojo negro, condición basal de la safena interna antes de la ablación- observada con Ecografía de Modo B - el del ojo ciego o blanco, en el corte transversal de la safena interna en el momento de la ablación y el del ojo gris, cuando examinamos con el ecógrafo en el postoperatorio inmediato. Los ecógrafos que utilizamos son los portátiles, Sonosite 180 Plus; Sonosite Titán y Sonposite Micromaxx. Nuestra experiencia es cercana a los 700 casos propios.

\section{Conclusión}

El Método Terapéutico Laser Endoluminal Percutáneo Ecoguiado ( LEPE), para el tratamiento de la vena safena interna incompetente, así también como de las venas perforantes y demás venas superficiales incompetentes ha demostrado ser eficaz, a lo largo de más de 10 años de experiencia que tenemos en nuestros grupos de trabajo. La efectividad ha sido comprobada al principio a los 3 y a los 6 meses, actualmente tenemos casos de 10 años de seguimiento, en los cuales, la oclusión de los vasos o la disminución del calibre es la regla. También se observa reacomodación anatómica y readaptación hemodinamica con mejoría clínica del paciente. El seguimiento clínico es de gran importanica, como en toda intervención quirúrgica. El Ecodoppler nos permite detectar precozmente si hay recidiva, la cual se trata con Foam Ecoguiado en la mayoría de los casos. Así también como las complicaciones, tener en cuanta la TVP, de muy baja incidencia, pero muy importante en caso que se presente en el Postoperatorio. Aconsejamos control ecográfico após 7 días.

Palabras-Clave: laser, fibra óptica, ecodoppler, varices. 